\title{
El rol estratégico de los gobiernos locales y regionales en la implementación de la Agenda 2030: experiencias desde la cooperación Sur-Sur y triangular
}

\section{Tahina Ojeda Medina*}

\section{RESUMEN}

Los gobiernos locales y regionales tienen un rol estratégico en la implementación de la Agenda 2030. Estos actores, a través de la cooperación Sur-Sur descentralizada y la cooperación triangular, cobran especial relevancia, tanto en la planificación multinivel como en la ejecución de políticas públicas destinadas a la implementación de los Objetivos de Desarrollo Sostenible en sus territorios. Con la revisión de distintas experiencias en América Latina se ponen de relieve sus aportaciones al desarrollo sostenible y a las alianzas multiactor en el contexto de la Agenda 2030. Se presentan estudios de caso sobre cooperación Sur-Sur descentralizada y cooperación triangular relacionados con la localización de los oDs.

Palabras clave: Agenda 2030, gobiernos locales, localización oDs, cooperación SurSur, cooperación descentralizada, cooperación triangular.

* Doctora en ciencia política. Investigadora del Instituto Universitario de Desarrollo y Cooperación de la Universidad Complutense de Madrid (España). Profesora de la Universidad Antonio Nebrija (España). [tojeda@iudc.es], [https://orcid.org/0000-0002-6012-465X].

Recibido: 3 de junio de 2019 / Modificado: 25 de julio de 2019 / Aceptado: 26 de julio de 2019

Para citar este artículo:

Ojeda Medina, T. (2020). El rol estratégico de los gobiernos locales y regionales en la implementación de la Agenda 2030: experiencias desde la cooperación Sur-Sur y triangular. OASIS, 31, pp. 9-29

DOI: https://doi.org/10.18601/16577558.n31.03 


\section{The strategic role of local and regional governments in the implementation of the 2030 Agenda: experiences from South-South and triangular cooperation}

\section{ABSTRACT}

Local and regional governments have a strategic role to play in the implementation of the 2030 Agenda. These actors, through decentralized South-South cooperation and triangular cooperation, take on special relevance both in multi-level planning and in the execution of public policies aimed at the implementation of the Sustainable Development Goals in their territories. The review of different experiences in Latin America highlights their contributions to sustainable development and multistakeholder partnerships in the context of the 2030 Agenda. Case studies on decentralized South-South cooperation and triangular cooperation related to the localization of the SDGs are presented.

Keywords: 2030 Agenda, local governments, sDGs location, South-South cooperation, decentralized cooperation, triangular cooperation.

\section{INTRODUCCIÓN}

Hasta ahora los Estados constituyen la fuente principal de planificación, financiación y ejecución de la política de Cooperación Internacional para el Desarrollo (CID), en todas sus modalidades. Además, protagonizan los espacios de concertación política y técnicos de cooperación. Ante esta realidad, los gobiernos locales y regionales (GLR) han quedado en un segundo plano en los estudios académicos, con escaso reconocimiento y visibilidad de sus prácticas de Css y CT.

Los GLR son agentes convocados e interesados en la implementación de la Agenda 2030 para Objetivos de Desarrollo Sostenible (oDs) y la Nueva Agenda Urbana. A través de la cooperación Sur-Sur (css) descentralizada y la cooperación triangular (Ст), estos actores cobran especial relevancia en la planificación multinivel y en la ejecución de políticas públicas destinadas a la implementación de los oDs. Esta labor ha quedado reconocida en la Segunda Conferencia de las Naciones Unidas sobre la Cooperación Sur-Sur (Paba+40).

La academia, en tanto actor convocado en el Paba+40, también está llamada a la implementación de la Agenda 2030. Por ello, desde la investigación aplicada, debe apoyar a todos los actores para fortalecer y promover su actuación en el campo del desarrollo sostenible. En el caso de los GLR, estudiar y conocer sus prácticas contribuye a comprender el rol estratégico que desempeñan en el logro de los ODS. El objetivo del presente artículo, por tanto, es analizar y visibilizar este rol estratégico a través de la css descentralizada y la CT, poniendo el foco en América Latina.

La hipótesis que aquí se plantea es que la amplia trayectoria que los GLR latinoamericanos tienen en la css descentralizada, e incipiente en la Ст, queda eclipsada por el exacerbado protagonismo dado a los Estados en la 
cooperación internacional. Sin embargo, son agentes del desarrollo sostenible que poseen características idóneas y experiencias relevantes para sumarse a las alianzas multiactor en la localización de los ODs en sus territorios. La css descentralizada y la ст se presentan como herramientas útiles para conseguirlo.

Por ello se presentan algunas experiencias de los GLR organizadas en dos grupos. El primero de ellos describe cómo se está dando el proceso de localización de oDs en la planificación del desarrollo sostenible en Colombia, México y Brasil bajo esquemas de política multinivel y con participación multiactor. Y el segundo, detalla dos proyectos que vinculan a GLr de Argentina, Chile, Uruguay, Paraguay y Brasil en procesos de css descentralizada y CT relacionados con oDs específicos.

Se empleó el método analítico con un enfoque cualitativo, desde el que se recopilaron e interpretaron testimonios, documentos y experiencias relacionadas con el objeto de estudio. Las fuentes primarias constan de informes de ejecución de proyectos de css descentralizada y Ст para la localización de los ODS, documentos de debate producidos en la Conferencia del Paba+40, noticias de diarios locales e informes de gestión de agencias de cooperación e instituciones públicas de Colombia, México, Argentina, Chile y Uruguay. Las fuentes secundarias constan de artículos académicos y publicaciones especializadas. Las técnicas de recolección de los datos cualitativos consistieron en: a) cuatro entrevistas semiestructuradas a dos informantes clave, Guadalupe Simón (Provincia de Salta, Argentina) y Lucy Larrosa (Gobierno de la Provincia de Cerro Largo, Uruguay); b) participación de la autora en diversos Side Events de la Conferencia del Paba+40 en Buenos Aires; y c) la revisión de documentos de instituciones públicas y organizaciones internacionales.

\section{GOBIERNOS LOCALES Y REGIONALES: EL TRÁNSITO DE LOS ODM A LOS ODS}

El 2015 supuso el plazo límite para dar cumplimiento a los Objetivos de Desarrollo del Milenio (ODM) y el cierre de un ciclo de políticas de desarrollo enfocadas a la reducción de la pobreza extrema y el hambre.

Del período 2000-2015 quedaron varias lecciones sobre la mesa. Sobre todo, las referidas a: el enfoque de necesidades básicas al que quedaron suscritos los odm; la falta de transparencia y participación en la selección de los ODM; la asimetría en la distribución de las competencias y responsabilidades -en los ámbitos locales, nacionales, regionales e internacionales-; las limitaciones en los métodos de medición de avances nacionales; las restricciones en la financiación para la implementación; el excesivo protagonismo de los Estados en la implementación de la agenda en detrimento de otros actores y agentes del desarrollo (Ojeda, 2015).

Los GLR tienen, entre otras características, competencias en la prestación de servicios de carácter básico; un papel determinante en la gestión de los flujos migratorios internos e internacionales -en la medida en la que gestionan políticas de integración socioeconómicas para las personas migrantes-; desempeñan un rol en la lucha contra el cambio climático por cuanto el $80 \%$ de las emisiones de $\mathrm{CO}^{2}$ se producen en entornos urbanos-; y tienen una 
larga trayectoria de trabajo en redes internacionales de cooperación (Fernándezy Del Huerto, 2016). A pesar de ello, solo los Estados y los gobiernos nacionales fueron los convocados para orientar e implementar las políticas de desarrollo que conducirían al logro de los 8 ODM y sus 49 metas.

Tras la experiencia de la implementación de los odm, la Agenda 2030 se gestó en un marco sensiblemente diferente en el que se evidenció:

a) El cuestionamiento activista y epistemológico de los modelos de desarrollo y de las formas de producción y consumo que le dan sustento.

b) El alto dinamismo de la css que “[... plantea desafíos a la concepción y gobernanza del sistema internacional y abre el debate sobre las formas de ayuda para definir nuevos consensos y nuevos actores" (Bárcenas, 2016, p. 16).

c) Lo limitados que fueron los ODM, en parte debido a que no lograron recoger las heterogeneidades de desarrollo existentes en el Sur. Esto, sumado a otros factores de tipo económico y político, llevó a los países del Sur a establecer otras agendas de trabajo que consideraron más ajustadas a sus intereses y necesidades de desarrollo y cooperación.

c) La nueva geografía política del desarrollo y de las relaciones Norte-Sur marcada por la diversificación de espacios de representación y de actores en el escenario internacional.

d) El incremento de la demanda de participación activa y significativa de los actores locales y regionales en los procesos de desarrollo y cooperación nacional e internacional.

Los actores locales y regionales no quedaron plenamente reconocidos en la distribución de competencias en la implementación de los oDM y tampoco reconocidas sus aportaciones y necesidades territoriales. Desde entonces, aumentaron y fortalecieron sus espacios de organización y participación internacional en sectores claves del desarrollo urbano y rural sostenible.

De cara a la Agenda 2030, a través de su trabajo de incidencia política y en red, los actores locales lograron: alcanzar el reconocimiento expreso de su papel como actores en el desarrollo sostenible, influir en la necesidad de dotar a los oDs de un enfoque territorial y establecer el oDs 11 sobre Ciudades Sostenibles y Asentamientos Urbanos.

Así, el diseño de los oDs y la Agenda 2030 presenta las siguientes características (GTLRG, ONU-Hábitat y PNUD, 2016, p. 14):

- Enfoque multidimensional del desarrollo sostenible (social, económico y ambiental).

- No dejar a nadie atrás: es para todas las personas.

- Carácter global: los ods abordan los retos globales más apremiantes de nuestro tiempo.

- Naturaleza universal: se aplica a todos los países del mundo independientemente de sus niveles de ingresos.

- Integralidad: es integral e interconectada en todas sus dimensiones y a todos los ni- 
veles: entre objetivos, entre países y entre niveles global, regional y nacional.

- Inclusiva: implica a todos los niveles de gobierno, a todos los actores interesados y a todas las personas en un esfuerzo inclusivo y colectivo para el desarrollo sostenible. Este elemento muestra a la Agenda 2030 como una asociación mundial para el desarrollo sostenible.

- Medible: pone un énfasis especial en la necesidad de medir el desempeño y los resultados a través de un conjunto de indicadores para evaluar el logro de los oDs y extraer lecciones y recomendaciones.

El período 2015-2030 abre, por tanto, un ciclo de políticas públicas de desarrollo con un enfoque holístico, de construcción de capacidades y de superación de la pobreza multidimensional, convocando a una alianza multiactor para el desarrollo sostenible. Para los GLR el trabajo cooperativo y coherente, en alianza con todos los actores territoriales de interés, será la clave para potenciar sus aportaciones al desarrollo sostenible.

\section{GOBIERNOS LOCALES Y REGIONALES ANTE LA AGENDA 2030, CSS Y CT}

La Agenda 2030 es una hoja de ruta que convoca a los actores, con independencia de su naturaleza, a poner en común esfuerzos, capacidades, conocimientos, financiación y políticas en favor del desarrollo sostenible global.
Comprende 17 ods, 169 metas y 241 indicadores marco ${ }^{1}$. Los oDs representan solo uno de sus componentes, ya que la Agenda supone un compromiso destinado a realizar cambios profundos en el sistema internacional. Alcanzar los ods "requerirá un cambio en el estilo de desarrollo y en las políticas económicas, industriales, sociales y ambientales en sintonía con un cambio estructural progresivo" (Bárcena, 2016, p. 16). Con las características que presentan, los oDs y la Agenda 2030 se configuran como un llamado a la acción global para atender los retos que enfrenta la sociedad internacional en su conjunto, de manera diferenciada y localizada.

Lograr las 169 metas también requiere emplear estrategias innovadoras, participación activa y significativa de todos los actores del desarrollo, así como implementar políticas públicas bien articuladas y coherentes en los distintos niveles de gobierno. Pero, sobre todo, requiere de un alto grado de cooperación y socialización de experiencias, recursos, tecnologías e información entre los actores clave en sus diferentes niveles de actuación.

Es aquí donde la Cooperación Internacional para el Desarrollo (CID), en todas sus modalidades y formas, tiene un papel destacado al poseer la cualidad de vincular a los actores que disponen de las capacidades, los conocimientos y los recursos necesarios para la implementación de la Agenda. Actualmente, gracias a la naturaleza universal y el carácter inclusivo que tienen los oDs, la css y cT se presentan como

\footnotetext{
1 Cuenta con el "Marco de indicadores mundiales para los Objetivos de Desarrollo Sostenible y metas de la Agenda 2030 para el Desarrollo Sostenible”, https://undocs.org/es/A/REs/71/313
}

OASIS, ISSN: 1657-7558, E-ISSN: 2346-2132, N³1, Enero - Junio de 2020, pp. 9-29 
modalidades de cooperación de especial utilidad para facilitar su implementación. Al punto que el oDs 17, "revitalizar la alianza mundial para el desarrollo sostenible", contiene dos metas que involucran directamente a la css y Ст (17.6 у 17.9).

La css, entendida como un entramado de relaciones y de intercambio cooperativo y complementario con objetivos de desarrollo entre actores del Sur, se fundamenta en la identificación previa de una serie de necesidades locales, nacionales o regionales. Estas dan origen a la solicitud de cooperación y a la negociación, tras la cual se determinarán los objetivos y las actuaciones mutuamente consensuadas entre las partes que establecen la relación (Ojeda, 2019).

Sus áreas de trabajo están directamente relacionadas con las necesidades identificadas en combinación con los objetivos de desarrollo nacional, muchos de ellos alineados con los ops. Lo que origina los intercambios en la css es la priorización de aquellos sectores y objetivos en los que las partes hayan adquirido alguna capacidad o experiencia que haya resultado de utilidad en un contexto similar.

[...] la relevancia de la [css] ha aumentado como una modalidad importante de la cooperación internacional para el desarrollo sostenible ya que su escala, su alcance y el número de agentes implicados han ido en aumento. [...]. La creciente importancia y contribución de la [css] a los objetivos de desarrollo convenidos internacionalmente ha sido impulsada por los importantes avances conseguidos por muchos países en desarrollo. En la actualidad, la [Css] contribuye a la transformación de los principios y normas de la cooperación internacional. [...]. La determinación mostrada por los interesados del Sur en la promoción de alianzas [...] pone de relieve la importancia de la [css] a la hora de hacer frente a los persistentes problemas de desarrollo y alcanzar los objetivos de la Agenda 2030 (onu, 2018, párr. 1, 4 y 6c).

Por su parte, la CT es

una modalidad que fomenta las alianzas y la confianza entre todos los asociados y que combina diversos recursos y capacidades, bajo la responsabilidad del país en desarrollo que la solicite, para alcanzar [los oDs]; y que aporta un valor añadido mediante el aprovechamiento y la movilización de recursos técnicos y financieros adicionales, el intercambio de una gama más amplia de experiencias, [...] y la combinación de soluciones de desarrollo asequibles y adaptadas a cada contexto [...] (UN, 2019, párr. 28).

En la css, al igual que sucede en la Norte-Sur, a pesar de que participan una amplia gama de actores y agentes, ha privilegiado al Estado y al gobierno nacional como sus protagonistas y promotores. Esto se explica por el carácter político e intergubernamental con el que nació la cid y su vinculación con la política exterior. El reconocimiento e incorporación de otro tipo de actores es producto de largos procesos de incidencia política y de experiencias que evidencian que también existe capacidad y potencial más allá del Estado.

En este sentido, se está prestando particular atención a los GLR en la CSS y la CT. Estos actores han conseguido, no sin esfuerzo, el reconocimiento a su labor en el desarrollo 
sostenible y su potencial para contribuir a la implementación de la Agenda 2030.

La Conferencia del Paba+40, celebrada en Buenos Aires del 20 al 22 de marzo de 2019, se centró en "El rol de la cooperación Sur-Sur y la implementación de la Agenda 2030 para el Desarrollo Sostenible: desafíos y oportunidades". En ella se reconoce el rol estratégico que cumplen los GLR en la implementación de la Agenda, tal y como se muestra a continuación (un, 2019):

\section{Actores y alianzas}

- $\quad$ Reconoce que la css ha aumentado el número de agentes que intervienen en el contexto del desarrollo, destacando a las entidades subnacionales, la sociedad civil y el sector privado (párr. 16).

- Indica que los gobiernos tienen el papel principal de coordinar y dirigir las iniciativas de desarrollo, pero insta a todos los actores y agentes de la cooperación a que tengan una participación significativa en ellas (párr. 22).

- Solicita se establezcan y promuevan soluciones y alianzas triangulares para el desarrollo innovadoras, en las que participen las autoridades locales y regionales, la sociedad civil, el sector privado, el mundo académico y ONG (párr. 28b).

- Alienta a los Estados Miembros a que establezcan o refuercen mecanismos de coordinación a nivel subnacional, nacional, regional y mundial para aprovechar los conocimientos especializados y otros recursos en apoyo a la Css y CT (párr. 31).

\section{Instrumentos}

- Cooperación técnica, intercambio de experiencias, conocimientos, formación, desarrollo de capacidades y transferencia de tecnología mutuamente convenida, entre otras (párr. 17).

- Intercambio de buenas prácticas en el ámbito de la planificación y la gestión urbanas participativas (párr. 32).

\section{Ciudades y gobiernos locales}

- Reconoce la tendencia a la urbanización rápida de los países en desarrollo y solicita que se intensifiquen las iniciativas de css у ст (párr. 32).

- Destaca la importancia de la css y ct en favor del trabajo decente, el desarrollo de las comunidades locales y la prestación de servicios en las zonas rurales para hacer frente a los factores que impulsan la migración del campo a la ciudad y a los problemas conexos (párr. 32).

\section{Mecanismos de coordinación}

- Alienta a los países a que adopten o refuercen políticas nacionales para promover la Css у CT, y a que aumenten la capacidad de los mecanismos nacionales y subnacionales de coordinación (párr. 24g).

- Exhortan a los centros de excelencia a que fortalezcan las plataformas Sur-Sur y de intercambio de experiencias entre las agrupaciones subregionales, con el propósito de establecer alianza entre múltiples 
interesados y programas de colaboración transfronterizos (párr. 24h).

La Agenda 2030 convoca a los GLR, junto a todos los actores interesados, a la localización de los oDs en las políticas de desarrollo en sus territorios. Es un buen momento para aprovechar las herramientas que brindan la css y Ст, tanto para intercambiar experiencias, conocimientos y recursos técnicos a favor del desarrollo sostenible, como para impulsar actuaciones conjuntas que deriven en políticas públicas de desarrollo con beneficios compartidos.

\section{GOBIERNOS LOCALES Y REGIONALES ANTE EL RETO DE LOCALIZACIÓN DE LOS ODS}

Localizar o territorializar los oDs se refiere al proceso de adaptación, implementación y monitoreo de los oDs a nivel local:

Es el proceso que tiene en cuenta los contextos subnacionales en el logro de la Agenda 2030, desde el establecimiento de los objetivos y metas, hasta la determinación de los medios de implementación, y el uso de indicadores para medir y monitorear el progreso hecho. La localización guarda relación tanto a cómo los gobiernos locales y regionales pueden dar apoyo para alcanzar los oDs a través de la acción "desde abajo", y cómo los ods pueden ofrecer un marco para la política de desarrollo local (GTLRG, 2016, p. 5).
La localización de los oDs supone que estos deben trasladarse al ámbito concreto de las políticas públicas de la manera más coherente y armonizada posible entre objetivos, agentes y agendas -en plural-. Por tanto, el momento es propicio para tomar en cuenta las siguientes consideraciones.

Primero, actualmente coexisten cinco agendas que resultan complementarias pues sus objetivos repercuten en el desarrollo sostenible: 1) Agenda 2030; 2) Nueva Agenda Urbana; 3) Acuerdo de París sobre Cambio Climático; 4) Marco de Sendái para la Reducción de Riesgos de Desastres; 5) Agenda de Acción de Addis Abeba. Sus preceptos y orientaciones no pueden ser asumidos de manera aislada ni pretender que se localice solo una de ellas. En conjunto suponen el actual marco internacional de orientación política para el logro del desarrollo sostenible en todos los niveles ${ }^{2}$.

Segundo, asumir la complementariedad de las agendas internacionales para la localización de los oDs repercutirá en la eficacia de los siguientes procesos: a) interconexión de objetivos en los ejercicios de priorización local; b) incrementar y coordinar la financiación -nacional e internacional- para su implementación; c) generar espacios de participación activa entre múltiples actores interesados; d) establecer criterios para los sistemas y mecanismos de recolección de datos locales e información de

2 La XI Asamblea General del Foro Mundial de Regiones (Rabat, 22-23 de octubre 2018) da un primer paso en proponer la convergencia de estas agendas de cara al proceso de implementación por parte de los GLR. Ver Declaración de Rabat (oru-Fogar, 2018) https://bit.ly/2y4M0мт. 
calidad; e) alineación de los planes locales de desarrollo territorial con los planes nacionales de desarrollo y planificación estratégica multinivel. Esta complementariedad cobra aún más sentido para los GLR si se toma en cuenta que la localización va más allá del ods 11 "lograr que las ciudades y los asentamientos humanos sean inclusivos, seguros, resilientes y sostenibles". La mayoría de los ods, así como el resto de agendas, contemplan metas relacionadas con las competencias de los GLR. Además, los desafíos a los que pretende hacer frente la Agenda 2030 tienen un claro impacto local y ningún oDs puede ser implementado de manera coherente y exitosa sin la participación activa y significativa de los GLR (Fernández, 2016).

Tercero, la educación para el desarrollo y la sensibilización de la ciudadanía resulta otro elemento clave en la localización de los oDs. "La sensibilización pretende [...] dar a conocer en principio la Agenda 2030 y los ODs, pero también la situación local y las razones por las cuales la Agenda se presenta como una oportunidad política para trabajar desde diferentes ámbitos y en articulación con diversos actores" (Agüero, 2019, p. 11).

Estas consideraciones bien pueden representar el punto de partida para iniciar cualquier ejercicio de localización de los oDs, en la medida que permiten orientar los diagnósticos de situación y necesidades locales, así como trazar la ruta que seguirán los planes de desarrollo territorial y las actuaciones de cooperación de los actores locales.

Una vez realizado este ejercicio se podrá avanzar en las cuestiones técnicas necesarias para la localización de los ops en los territorios.
No sin antes recordar cuestiones elementales como:

- Coherencia: las políticas públicas nacionales y locales, así como los planes de acción deben estar en alineación con los oDs, contar con presupuestos coherentes para su implementación y disponer de sistemas de monitoreo y seguimiento. Esto permitirá identificar dónde están los principales aceleradores para el cumplimiento de varios oDs, conectándolos con las otras agendas globales.

- Participación y diálogo horizontal: que permita establecer vínculos de confianza $\mathrm{y}$ formas eficaces de trabajo colectivo con el elenco de actores locales.

- Adecuación de marcos normativos: que actúen más como aceleradores que obstáculos a las políticas públicas y planes de acción en todos los niveles de gobierno, al tiempo que faciliten la css y ст.

- Sistemas de registro, monitoreo y evaluación: con el fin de que los datos generados sean útiles es necesario crear, adecuar y fortalecer (según sea el caso) estos sistemas -locales y nacionales- para el seguimiento al cumplimiento de las metas y a la ejecución interconectada de los objetivos.

- Indicadores propios: la creación de estos indicadores es fundamental ya que "no solo permiten el seguimiento y la revisión a nivel territorial, sino que también contribuyen a las revisiones voluntarias nacionales, proporcionando aportes relevantes para los esfuerzos de monitoreo nacionales y mundiales" (Messias, Grigorovski y Sindico, 2018, p. 2). 
LA CSS DESCENTRALIZADA Y CT COMO HERRAMIENTAS ÚTILES PARA

LAS INICIATIVAS DE LOCALIZACIÓN DE LOS ODS EN AMÉRICA LATINA

Los GLR poseen un valor añadido para la localización de oDs por su actuación en el campo de la CID. Emplean mecanismos de trabajo conjunto e instrumentos prácticos que reportan un doble dividendo. Por un lado, facilitan el intercambio de conocimientos y experiencias con otros GLR para la resolución de problemas concretos $y$, por otro, construyen alianzas que permiten ejercer presión para la toma de decisiones en los ámbitos nacional e internacional (Ojeda, 2019).

La css descentralizada es una forma específica de ejecución de la css. Representa una práctica habitual en la relación entre los GLR que favorece espacios de encuentro para fortalecer las capacidades de instituciones locales, ganar espacios de decisión política y visibilidad, promover soluciones compartidas a problemas comunes, diversificar y ampliar las fuentes de financiación, transferencia de buenas prácticas en sectores y objetivos focalizados, etc.

A día de hoy, las vías de canalización de la css descentralizada entre GLR en América Latina son principalmente bilateral y subregional. No obstante, también se están registrando experiencias vía $\mathrm{CT}$, así como con participación de la sociedad civil y del sector privado.

La bilateral, que es la más utilizada, se realiza directamente entre los GLR. Su puesta en marcha, por lo general, sucede por cuestiones tan dispares como la identificación de necesidades y/o problemas comunes, la identifica- ción de capacidades y experiencias relevantes que, tras su conocimiento, resultan de utilidad en otro territorio, la voluntad política de las autoridades locales en momentos concretos o, sencillamente, por el conocimiento y la confianza mutua entre los gestores locales de la cooperación.

La subregional, menos utilizada, responde al trabajo en red de los GLR. Se ejecuta predominantemente por gobiernos -aunque también por otros actores no estatales-a través de su participación en redes de distinto tipo: integración fronteriza de carácter subregional, regionales de municipalidades, temáticas dentro de esquemas de integración regional, internacionales de gobiernos locales y municipalidades, académicas, entre otras.

\section{EXPERIENCIAS DE LOCALIZACIÓN DE ODS EN AMÉRICA LATINA}

\section{Colombia: Alineación de los Planes de Desarrollo} Territoriales (PDT) a los ODS en el periodo 20162019. El país cuenta con la Comisión Interinstitucional de Alto Nivel para el Alistamiento y Efectiva Implementación de la Agenda 2030 y sus ods (Comisión ods). Dicha Comisión está presidida por el Departamento Nacional de Planeación (DNP) y prevé la participación de actores de la academia, el sector privado, la sociedad civil y medios de comunicación.

El Dnp diseñó el Kit Territorial para dar apoyo técnico a los GLR que "[...] contenía las metodologías, herramientas y orientaciones para la formulación de los [PDT], además de los lineamientos [...] con los ODs" (DNP, 2017, p. 31). Además, para agilizar el proceso de orientación de los oDs en las planificaciones terri- 
toriales y los PDT, realizó reuniones a través de videoconferencias con los equipos de planeación de las gobernaciones departamentales y de las alcaldías de las ciudades capitales del país.

Cuando los PDT 2016-2019 estuvieron aprobados por las autoridades competentes, la Secretaría Técnica de la Comisión ods, representada por la Dirección de Seguimiento y Evaluación de Políticas Públicas (DSEPP) del DNP, realizó un estudio para constatar el grado de alineación de los Planes de Desarrollo Departamentales (PDD) con los ods. Dicho estudio comprendió tres fases: 1) Identificar el compromiso político con los oDs en los PDD; 2) Identificar inclusión de los ods en el componente estratégico de los PDD y; 3) Análisis técnico y síntesis.

Los resultados fueron clasificados por la DNP en las siguientes categorías: general, medio y alto.

[...] la incorporación general, algunos PDD incluyeron los oDs como referencia, pero sin priorizar o establecer una asociación explícita entre los componentes estratégicos del plan de desarrollo y su contribución al cumplimiento de los oDs. A nivel medio, además de hacer referencia a los ODs, los PDD precisaron la relación entre los mismos y los grandes pilares, dimensiones o ejes estratégicos de los PDD; sin embargo, no fue evidente la asociación explícita entre programas, subprogramas, objetivos programáticos o metas concretas del PDD con las metas oDs. Por último, $[\ldots]$ en los que se observó una alta incorporación de los ODS, estos se incorporaron tanto en las partes generales del documento como en algunos de sus componentes estratégicos, incluyendo la asociación explícita entre los ods (a nivel de objetivo o meta) con programas, subprogramas, metas o indicadores específicos (de resultado o de producto) de los PDD (DNP, 2017, p. 44).

México- Estrategia del Gobierno Federal para una institucionalización nacional de la Agen$d a$, que integre a las autoridades subnacionales. La Oficina de la Presidencia de la República (Opr), la Comisión Ejecutiva para el Cumplimiento de la Agenda 2030 y el Instituto Nacional para el Federalismo y el Desarrollo Municipal (Inafed), promovieron la creación de los Órganos de Seguimiento y Monitoreo (osI) como "espacios de organización mediante el cual los gobiernos estatales se suman a los esfuerzos nacionales para la puesta en marcha de los oDs, intercambian experiencias, coordinan acciones y desarrollan mecanismos de monitoreo y seguimiento" (PNUD, 2019, p. 13).

Entre las actividades destacan: a) priorización de 14 ods y 25 metas en función de las competencias legales de los gobiernos municipales; b) aplicación de un modelo de asistencia técnica, por parte de Inafed, para dar seguimiento a la implementación de los oDs en los gobiernos municipales; c) sensibilización y capacitación de funcionarios municipales sobre la Agenda 2030. Por su parte, la opr elaboró la "Guía para Incorporar el Enfoque de la Agenda 2030 en la Elaboración de Planes Estatales y Municipales de Desarrollo"3, como forma de

3 Véase https://bit.ly/2wjhldQ 
orientación técnica a la elaboración de políticas públicas locales alineadas con los oDs.

Brasil: el caso de la Región Metropolitana de Belo Horizonte. La necesidad de avanzar en la generación de datos e indicadores de seguimiento de la implementación de los oDs, tanto a nivel nacional como regional, potenció en Brasil varias alianzas multiactor. A nivel nacional y regional resultan especialmente relevantes los trabajos del Grupo de Trabajo de la Sociedad Civil Brasileña para la Agenda 2030 y del Observatorio Metropolitano de ods (Metrods).

En Belo Horizonte se impulsó en 2017 el proyecto ODS Em Ação, en el que participan Metrods, la Universidad Newton Paiva y el Movimento Nossa BH, con apoyo financiero y técnico del Thematic Research Network on Data and Statistics (Trends) de Estados Unidos.

Según el informe de TRends (2019) la Universidad aportó profesorado experto en diversas áreas del desarrollo sostenible y estudiantes que apoyaron al gobierno de Belo Horizonte a desarrollar una base de datos para los oDs y su recolección de forma participativa. El Movimento Nossa BH aportó su experiencia en producción y análisis de datos para revisar la información que se recolectó en las ciudades de Belo Horizonte. Metrods desarrolló un marco de 80 indicadores, en su mayoría para el oDs 11, y movilizó a sus instituciones afiliadas para difundir los resultados y las cifras que surgieron del trabajo desarrollado en el marco del proyecto. La coordinación de los enlaces y reuniones entre los gobiernos locales de Belo Horizonte y las instituciones miembros del proyecto $O D S$
Em Ação contó con el apoyo de la Agencia de Desarrollo de la Región Metropolitana de Belo Horizonte, que además colaboró con el Metrods en la prueba piloto de su metodología de monitoreo y evaluación participativa y el marco de indicadores del oDs 11.

Las experiencias antes presentadas dan muestra del incipiente nivel de articulación de políticas de tipo multinivel y del diálogo multiactor necesario para la implementación del EIDs que promueve la Agenda 2030. En ese sentido, se observa que cada experiencia refleja las particularidades del contexto local y nacional, relacionado tanto con las necesidades identificadas por los actores como con las lógicas de trabajo institucional, normativo y organizacional con las que se relacionan los actores nacionales. Si bien existen muchas más experiencias, las de Colombia, Brasil y México contienen elementos significativos de innovación (p. ej. planificación, actores e instrumentos) que pueden resultar útiles para el intercambio de experiencias en materia de localización de oDs por parte de los GLR a través de la css descentralizada у ст.

\section{Experiencias de css descentralizada y CT relacionadas con oDs}

Proyecto Mirada Ciudadana: la buena gobernanza en municipios del Mercosur. Alineación con oDs: 11,16 y 17 (principales), 8 y 9 (complementarios), 4 y 5 (transversales).

Nace como una iniciativa de css descentralizada entre gobiernos locales de Argentina, Chile, Brasil, Paraguay y Uruguay. Todos ellos 
miembros del Mercosur ${ }^{4}$, salvo el caso de Chile que es un Estado Asociado. Más en concreto, participan 17 municipios de las siguientes provincias: Los Andes y Peñalolén (Chile), San Isidro (Argentina), Aceguá y Candiota (Brasil), San Bernardino y Presidente Franco (Paraguay) y 9 municipios de Cerro Largo 5 (Uruguay), siendo la Intendencia de Cerro Largo la que actúa como coordinadora general del proyecto.

El proyecto se presentó a la convocatoria de financiación de cT de la Unión Europea a través del Programa Adelante en 2016, siendo seleccionado para 2016-2019. A partir de entonces, se consolida como un proyecto de CT entre 17 municipios del Mercosur y la Unión Europea.

La distribución de roles y responsabilidades acordadas entre las partes se organizó de la siguiente manera (Larrosa, 2019):

a) Cada municipio aporta la infraestructura y la logística para la realización de las actividades del proyecto, además de un funcionario/a técnico/a como enlace responsable en su territorio. También provee personal técnico especializado para la realización de los intercambios de experiencias y de conocimientos. b) La Intendencia de Cerro Largo aporta una funcionaria técnica coordinadora general del proyecto a tiempo completo, un contador, una secretaria y un técnico de comunicación a tiempo parcial. Además, contrata un técnico y un administrador a tiempo completo.

c) El Programa Adelante financia con 407.166,41€ y apoya en la gestión técnica y financiera, en la comunicación y visibilidad, en la participación y posicionamiento en eventos internacionales ${ }^{6} \mathrm{y}$ en la reflexión sobre la contribución del proyecto a los objetivos de desarrollo de la región. Adicionalmente, financia misiones de expertos internacionales para apoyar en aspectos puntuales del proyecto, como la localización de la Agenda 2030.

El proyecto se centra en el fortalecimiento de las capacidades de los gobiernos locales y de sus administraciones, así como de la sociedad civil, ante la necesidad de acordar un modelo de transparencia, rendición de cuentas y monitoreo ciudadano en la planificación y prestación de servicios públicos. Pretende "contribuir a que los Municipios del cono sur actúen eficientemente con la activa participación de

4 Estos gobiernos poseen relaciones previas de cooperación con ocasión de su participación institucional en el Foro Consultivo de Municipios, Estados Federados, Provincias y Departamentos del Mercosur (FCCR) y Mercociudades. No obstante, el proyecto no está adscrito a ninguna línea de trabajo dentro del Mercosur.

5 Aceguá, Noblía, Plácido Rosas, Río Branco, Tupambaé, Ramón Trigo, Arbolito, Fraile Muerto, Arévalo y Río Branco.

6 Los Días Europeos del Desarrollo (EDD), junio 2018, Bruselas. Global South South Development Expo, noviembre 2018, Nueva York. Conferencia Paba+40, marzo 2019. 
los ciudadanos, mejorando la gobernanza y la ejecución de políticas públicas de calidad para reducir las brechas de desigualdades existentes en la región y el logro de los oDs" (Intendencia de Cerro Largo, 2019).

El proceso de formulación y de ejecución de este proyecto es en sí mismo un ejercicio de cooperación descentralizada y de localización de ODS, a través del cual los gobiernos locales construyen, de manera colectiva y coordinada, sus propios mecanismos de trabajo, adaptados a sus necesidades y capacidades.

Se comenzó con la realización de un diagnóstico de necesidades de los municipios que sirvió de base para la formulación del proyecto. A pesar de las significativas diferencias institucionales, normativas ${ }^{7}$, culturales, sociales y financieras entre los 17 municipios, estos lograron identificar necesidades comunes. Entre ellas, destacan las de fortalecer las capacidades institucionales y de gestión pública local, asentar en las instituciones dichas capacidades para hacer frente a la rotación del funcionariado ${ }^{8}$ y articulación con actores de la sociedad civil y empresas.

Lucy Larrosa (2019), coordinadora general del proyecto, destaca que la metodología de trabajo empleada para adecuar las actividades del proyecto a las necesidades concretas de cada municipio, se orienta en dos líneas: establecer el diálogo político y fortalecer las capacidades de los municipios?.

Para establecer el diálogo político se realizan encuentros con las máximas autoridades políticas locales y en ellos se definen cuáles serán los temas de la agenda de cooperación. Estos diálogos pretenden identificar las necesidades específicas de cada gobierno local y definir las agendas de trabajo comunes en función de los temas identificados. Además, se definen los participantes de las acciones de cooperación y las temáticas de las actividades. Hasta la fecha se han realizado seis encuentros de diálogo político ${ }^{10}$ (Gobierno de Cerro Largo, 2019a y 2019b).

Para definir las actividades de fortalecimiento de las capacidades locales se cumplen las siguientes etapas: 1) realización de una entrevista a la autoridad del municipio para conocer cuáles son sus necesidades; 2) priorización de necesidades con los equipos de gestión técnica municipal y actores sociales; 3 ) diseño y adecuación de las actividades y módulos formativos a las necesidades de cada municipio. Estas tres etapas permiten "elaborar el traje a medida, es decir, no se repite la misma experiencia en cada municipio" (Larrosa, 2019). Hasta la fecha se han realizado 47 actividades

\footnotetext{
países.

Se refiere tanto al marco jurídico -nacional y local- como a las competencias atribuidas a cada GLR en los cuatro 8 Situación habitualmente producida por los cambios de gobierno en cada legislatura.

$9 \quad$ Primero tiene lugar el diálogo político y luego las actividades de fortalecimiento institucional de los gobiernos locales y la sociedad civil.

10 En 2017: Brasil - Jaguarón (1), Paraguay - San Bernardino y Presidente Franco - (2) y Argentina - San Isidro (1). En 2018, Paraguay - Alto Paraná (1) y Argentina - San Isidro (1).
} 
para fortalecer la gestión pública local y la atención ciudadana, en forma de talleres, reuniones, seminarios ${ }^{11}$, jornadas de planificación estratégica y encuentros temáticos (Gobierno de Cerro Largo, 2019a y 2019b).

Mirada Ciudadana está alineado con los oDs 11,16 y 17 (principales), 8 y 9 (complementarios), 4 y 5 (transversales). Dentro de las actividades de localización aplicables a los 17 municipios destacan tres líneas concretas: 1) incorporación de acciones sensibles al género en los presupuestos y políticas públicas de los municipios, con focalización en las mujeres afrodescendientes; 2) elaboración del "Manual para la implementación del sistema de información en cada municipio para la implementación y monitorear políticas públicas y oDs"; y 3) realización de un taller sobre "ods y su implementación a nivel local".

En el caso de los 9 municipios de Cerro Largo (Uruguay), con el apoyo de la Oficina de Planeamiento y Presupuesto de este país, se realizaron reuniones con alcaldes(as), concejales y otros actores locales para revisar y alinear las planificaciones quinquenales con los oDs. Esto incluyó la revisión de la planificación territorial para el quinquenio, el nivel de ejecución hasta 2018 y la identificación de los desafíos frente al horizonte 2030. Este ejercicio dio lugar a la preparación de un módulo de capacitación específico de formación sobre localización de los oDs y a la elaboración de una guía para localización de oDs en los municipios.
Proyecto Concientización sobre el uso responsable, eficiencia energética y gestión de la energía para un cambio cultural y de hábitos de consumo permanente y sostenible. Alineado con los oDs 7, 11 y 17.

Los gobiernos de la Provincia de Salta (Argentina) y de la Región de Antofagasta (Chile) mantienen una estrecha relación de css descentralizada y transfronteriza desde hace décadas. En los últimos años han identificado la necesidad de compartir experiencias y de fortalecer las capacidades para la generación de energías renovables y la gestión eficiente de la energía, contribuyendo así al desarrollo sostenible de ambos territorios y a la localización de los oDs.

Entre 2015-2017 se realizó el proyecto "Intercambio de experiencias en generación eléctrica distribuida”. Estuvo financiado por el Fondo Argentino de Cooperación Sur-Sur y Triangular (Foar) y por la Agencia Chilena de Cooperación Internacional para el Desarrollo (Agcid) y coordinado por la Representación de Relaciones Internacionales (RRI) del Gobierno de la Provincia de Salta y la Unidad Regional de Asuntos Internacionales (Urai) del Gobierno de la Región de Antofagasta. Participaron instituciones públicas de ambas regiones junto a actores sociales y privados locales.

Las partes coincidían en la necesidad de avanzar en el uso de energías renovables para contribuir a mejorar la eficiencia de gestión de los recursos en sus territorios. Chile había ternacional Gobierno Abierto y Gobernanza, Peñalolén (Chile), 11 al 14 de diciembre de 2018. 
desarrollado una iniciativa denominada Generación Ciudadana consistente

[...] en que los usuarios cuenten con equipamiento de generación de energías renovables y la cogeneración de electricidad [para] inyectar la energía excedente al sistema de distribución. Aprovechando la experiencia de Antofagasta de casi 10 años, se busca adaptar los conocimientos y procedimientos chilenos a las necesidades de Salta, fortaleciendo los recursos humanos de la Secretaría de Energía de Salta y el Ente Regulador de Servicios Públicos a través de capacitaciones que buscan diversificar la matriz energética, buscando contribuir al desarrollo sostenible de la población y al cuidado del medio ambiente (MREC, s.f.).

Para ambos gobiernos regionales el proyecto "Intercambio de experiencias en generación eléctrica distribuida", es considerado como el antecedente directo del proyecto "Concientización sobre el uso responsable, eficiencia energética y gestión de la energía para un cambio cultural y de hábitos de consumo permanente y sostenible" -en adelante, proyecto Eficiencia Energética-, según indica Guadalupe Simón, asesora técnica de la RRI de Salta.

Eficiencia Energética se formuló de manera conjunta entre los equipos técnicos de Salta (RRI y Secretaría de Energía del Gobierno de la Provincia) y Antofagasta (Urai y Secretaría Regional Ministerial de Energía del Gobierno de la Región). Fue presentado a la "Convocatoria para la presentación de proyectos de cooperación descentralizada Argentina-Chile 2017", y aprobado en la Comisión Mixta Argentino-
Chilena (5 y 6 de octubre de 2017) para su realización, en un período de 12 meses, entre 2018 y 2019. Culminaron sus actividades en junio del presente año.

Tiene como objetivos

Fortalecer la política energética de la provincia, contribuyendo al cuidado ambiental y al desarrollo sostenible mediante la incorporación de la eficiencia energética y la gestión de la energía. Implementar acciones concretas de concientización sobre el uso responsable y de energía para un cambio cultural y de hábitos de consumo permanente y sostenible en el tiempo sobre la base de la experiencia chilena (Agcid, s.f.).

Esta experiencia de css descentralizada cuenta con un conjunto de alianzas multiactor. Por Argentina participan: la RRI de Salta, el Ministerio de Ambiente y Producción Sustentable, la Secretaría de Energía, la Universidad Católica de Salta, la Empresa Distribuidora de Electricidad de Salta S.A. (Edesa), la Empresa Distribuidora de Gas Natural (Gasonor), la Compañía Salteña de Agua y Saneamiento, la empresa Aguas del Norte y el Copaipa. Por Chile participan: La URAI de Antofagasta, la Secretaría Regional Ministerial de Energía y el Ministerio de Energía de Chile.

La distribución de roles y responsabilidades acordadas entre las partes se organizó de la siguiente manera (Simón, 2019):

a) La aportación dineraria estaba estipulada en la "Convocatoria para la presentación de proyectos de cooperación descentra- 
lizada Argentina-Chile 2017"12. La Agcid financia con ocho millones de pesos chilenos, transferidos al Gobierno de la Región de Antofagasta tras la suscripción de un Convenio de Colaboración Interinstitucional. La Dirección General de Cooperación Internacional (DGCIN) del Ministerio de Relaciones Exteriores y Culto de Argentina (MREC), a través del Foar, aporta los pasajes, los viáticos y el seguro médico para los expertos que se desplazan para ejecutar las actividades previstas.

b) La RRI de Salta aporta tres funcionarios técnicos que realizan las gestiones de coordinación de las actividades del proyecto, así como la articulación con los actores locales y los aspectos técnicos de energía. También aporta las infraestructuras y la logística para las actividades (visitas a terreno, capacitaciones, reuniones y jornadas).

c) La Urai de Antofagasta aporta un funcionario técnico que trabaja en coordinación con un funcionario de la Secretaría de Energía. Desde la Urai se gestiona la preparación de las capacitaciones y la identificación de los expertos en las diversas instituciones públicas y privadas de Antofagasta. También se gestionan los recursos económicos del proyecto y se realiza el seguimiento de su ejecución.

d) La Universidad Católica de Salta y las empresas de suministro de energía, agua y gas, participan como receptores de las capacitaciones impartidas por los técnicos chilenos. Además, forman parte de las reuniones técnicas para la realización de una hoja de ruta o mapa de eficiencia y gestión de energía para la provincia.

Hasta la fecha se han realizado tres misiones en terreno, una en Antofagasta y dos en Salta (Simón, 2019).

La primera misión fue exploratoria. Una técnica y el director general de energía eléctrica de Salta presentaron el estado de situación de la gestión de energía y las necesidades identificadas en el territorio, con el fin de que los técnicos de Antofagasta pudiesen organizar los contenidos de las capacitaciones a impartir en las dos misiones restantes. Estas necesidades estaban relacionadas con los suministros a nivel mayorista y minorista de electricidad, gas $\mathrm{y}$ agua potable.

Posteriormente participaron en reuniones con representantes de diversas instituciones de Antofagasta para el intercambio de experiencias de gestión eficiente de energía y construcción sostenible de viviendas. Con ello se buscaba conocer la experiencia chilena en la implementación de planes, campañas y programas que servirían para definir los contenidos del programa de capacitación. Fueron invitados al Seminario Internacional "Emprendimiento e innovación - energías renovables no convencionales", 19 de octubre de 2018, donde Salta presentó la experiencia de alianza

12 Véase https://bit.ly/2wawlf4 
público-privada "Redes Eléctricas Inteligentes" del Barrio Grand Bourg de Salta Capital.

La segunda y tercera misión se centraron en la capacitación de los actores locales -públicos, sociales y privados- de Salta. Los técnicos de Antofagasta impartieron los módulos de capacitación en los temas solicitados por Salta a todos los actores participantes del proyecto.

Por último, precisar que el proceso de localización de los oDs para los proyectos de cooperación y desarrollo local de Salta se coordina a través de la Secretaría de Financiamiento. Dicha Secretaría, desde 2016, es responsable del desarrollo, adecuación, seguimiento y monitoreo de las metas de ods en Salta, de conformidad con el Convenio de Cooperación celebrado entre el Consejo Nacional de Coordinación de Políticas Sociales y la Provincia de Salta ${ }^{13}$ (CNPS, 2017, p. 18). También cuentan con el apoyo del equipo Interministerial para el desarrollo, adecuación, seguimiento y monitoreo de las metas de los ODs en la provincia. Actualmente siguen trabajando en la alineación de los diversos marcos de planificación provincial a los $\mathrm{ODS}^{14}$.

Las dos experiencias presentadas en este apartado reflejan la atención que los GLR de Argentina, Chile, Brasil, Paraguay y Uruguay están dando a los oDs y a la Agenda 2030. Muestran esfuerzos innovadores de articulación de políticas públicas en el ámbito subregional y el aprovechamiento de las herramientas de la css descentralizada y ст para fortalecer capa- cidades de los GLR en sectores específicos del desarrollo sostenible. Además, al igual que las experiencias de Colombia, México y Brasil, también representan oportunidades de intercambio de experiencias y conocimientos útiles para la gestión del desarrollo sostenible en el marco del oDs 17.

\section{CONCLUSIONES}

El objetivo central de este artículo fue analizar y visibilizar el rol estratégico de los GLR en la implementación de la Agenda 2030, a través de la css descentralizada y la CT, poniendo el foco en América Latina. Para ello, se estudió el marco internacional en el que progresivamente se ha ido fraguando el reconocimiento explícito de los GLR como agentes del desarrollo sostenible. Y, se explicó, a través de experiencias, el trabajo que vienen desarrollando para localizar los ods e implementar la Agenda 2030.

En las experiencias presentadas se enfatizaron los sistemas de alianzas y la distribución de tareas entre los distintos actores, debido a que el conocimiento de dichas prácticas puede resultar de utilidad y estímulo para el diseño de fórmulas innovadoras de trabajo en entornos de múltiples actores y sectores del desarrollo.

Vistos los casos de Colombia, México, Brasil, Chile, Argentina, Brasil, Paraguay y Uruguay, junto a otros que no han podido quedar reflejados en este texto, se muestra que los GLR poseen características idóneas y expe-
14 Ver Informe de ods Provincias (CNPS, 2017). 
riencias relevantes para sumarse a las alianzas multiactor en la localización de los oDs en sus territorios. Esto, sumado a su dilatada trayectoria en la css descentralizada, e incipiente en la CT, convierte a los GLR en un objeto de estudio relevante que debe ser valorado y reconocido también por la academia.

Cabe, pues, plantear una serie de cuestiones para el necesario estudio y debate académico a partir de ahora:

- La Agenda 2030 acerca lo local a lo global y, por tanto, es una tarea política de primer orden reconocer y visibilizar el rol estratégico que tienen los GLR en su implementación y en la CID (Norte-Sur, Sur-Sur y Triangular) para fortalecer sus espacios de acción territorial.

- Los gLR en América Latina deberán sortear retos internos, locales y nacionales, para hacer frente a los compromisos que asumen en la implementación de la Agenda 2030. Entre ellos: fortalecer capacidades institucionales y de gestión, alinear las planificaciones locales con las nacionales en el marco de los ods, contar con los recursos adecuados para las actuaciones que ponen en marcha, generar y sistematizar datos que reporten utilidad a las mediciones nacionales de cumplimiento de los oDs, trabajar con otros actores locales sociales y privados, etc.

- Hacer uso de la css descentralizada, como su propio nombre lo indica, no debe suponer su desconexión con la política nacional de css, pues no es sino una de sus formas de ejecución.

- El nivel de articulación de lo local con lo nacional es aún incipiente en muchos países de América Latina. Por tanto, es menester avanzar en la consolidación de los sistemas nacionales de desarrollo y de cooperación con la participación activa y significativa de todos los actores. Esto supone, como mínimo, por un lado, la revisión y actualización de los marcos institucionales y normativos para la adecuada distribución de competencias, presupuestos y responsabilidades. Por otro, conocer y reconocer las formas de trabajo de los diversos actores para idear fórmulas de actuación mutuamente convenidas, que respondan a la realidad y que apunten a objetivos comunes.

Finalmente, señalar que los GLR están incrementando su peso como actores en los campos del desarrollo y la css, ampliando sus espacios políticos y de actuación. Se espera de ellos que, en su escala, no repitan los errores derivados del exacerbado protagonismo de los Estados en la css. Se debe tener presente que en lo local existen multitud de actores de muy diverso tipo y capacidades. No se puede olvidar que la función principal de los GLR es la de servir de bisagra y punto de encuentro para articular procesos y actores. Dejar de mirarse de reojo y mirar juntos a su objetivo común puede ser un buen comienzo y la Agenda 2030 resulta la excusa perfecta para hacerlo. 


\section{REFERENCIAS}

Agencia Chilena de Cooperación Internacional para el Desarrollo (Agcid). (s.f.). Ficha resumen de nuevas iniciativas de cooperación descentralizada. Chile: Agci. https://bit.ly/2Mivjt4

Agüero, B. (2019). Procesos de localización de la Agenda 2030. Un estudio comparativo de municipios españoles. Madrid: Incidem.

Ayuso, A. y Forero, M. (2017). Objetivos de Desarrollo Sostenible: la agenda 2030 del compromiso a la práctica. España: сіDoв. https://bit.ly/2HiSawY

Bárcenas, A. (2016). Iberoamérica frente al desafío del desarrollo sostenible: una mirada desde la Cooperación Sur-Sur. Seminario Internacional. 13 y 14 de abril. Chile: PIFcss. Disponible en https:// bit.ly/2ogoucr

Celata, F.; Coletti, R. y Sanna, V. (2013). La cooperación transfronteriza en la región del Trifinio y la difusión de modelos europeos de gobernanza de las fronteras en América Latina. Si Somos Americanos. Revista de Estudios Transfronterizos, vol. 13, No. 2, diciembre. Chile: Universidad Arturo Prat, pp.165-189.

De la Fuente, R. (coord.) (2010). Los nuevos actores en la cooperación internacional. El papel de los gobiernos locales y regionales. Madrid: IUDC-UCM y Catarata.

Departamento Nacional de Planeamiento (DNP). (2017). Inclusión de los Objetivos de Desarrollo Sostenible en los planes de desarrollo territoriales, 2016-2019. Bogotá: Secretaría Técnica de la Comisión Interinstitucional de Alto Nivel para los ods en Colombia. https://bit.ly/2HkOeio

Dirección General de Cooperación Internacional (DGCIN) (s.f.). Cooperación Descentralizada Argentina. Una construcción conjunta como aporte para el desarrollo territorial. Buenos Aires: Ministerio de Relaciones Exteriores y Culto. https://bit. ly/2usnowU
Fernández, A. (2016). La cooperación descentralizada en Iberoamérica. Una mirada desde la cooperación SurSur. DT No. 11. Madrid: PIFCSS y Segib.

Fernández, A. y Del Huerto, M. (2016). Los gobiernos locales en la agenda internacional: ¿actores o espectadores? Cuadernos para la Internacionalización de las Ciudades, No 7. Gobierno de la Ciudad de México e Intendencia de Montevideo: AL-LAS.

Global TaskForce of Local and Regional Governments (GTLRG). (2016). Guía para la localización de los ODS: implementación y monitoreo subnacional. https://bit.ly/2E3O7Gt

GTLRG, ONU-Hábitat y PNUd. (2016). Primer módulo de capacitación sobre localización de los ODS. https:// bit.ly/2JmnfUe

Gobierno de Cerro Largo. (2019a). Primer informe descriptivo intermedio, Proyecto Mirada Ciudadana. Uruguay: Intendencia.

Gobierno de Cerro Largo. (2019b): Segundo informe descriptivo intermedio, Proyecto Mirada Ciudadana. Uruguay: Intendencia.

Consejo Nacional de Coordinación de Políticas Sociales (CNPS). (2017). Informe ODS Provincias. Proceso de adaptación. Argentina: Presidencia de la República. https://bit.ly/2vryO3H

Intendencia de Cerro Largo. (2019). Proyecto Mirada Ciudadana. Uruguay. https://bit.ly/2yThJfg

Messias, R.; Grigorovski, J. \& Sindico, F. (2018). Report Localizing the SDGs: Regional governmentes paving the way. Brussels: Network of Regional Governments for Sustainable Development (NRG4SD) \& University of Strathclyde Centre for Environmental Law and Governance.

Méndez, J. y Soto, W. (2015). Cooperación Sur-Sur en Centroamérica: Los casos de Trifinio y de los mecanismos de asistencia mutua ante desastres. Anuario de la Integración Regional de América Latina y el Caribe. No. 11. Buenos Aires: Cries, pp.171-186. 
Ministerio de Relaciones Exteriores y Culto (MREYC). (s.f.). Fortalecer capacidades en generación distribuida con fuentes renovables en redes de distribución. Argentina. https://bit.ly/2wuclmR NRG4SD. (2018). Gobiernos intermedios y la Agenda 2030 experiencias de territorialización de los oDs en América Latina. Seminario web. 18 de octubre.

Ojeda, T. (2018). La cooperación Sur-Sur transfronteriza como herramienta para la implementación de la Agenda 2030 y los ods, en L. Bendelac y M. Ramírez (coords.), La cooperación transfronteriza para el desarrollo (pp. 47-60). Madrid: IUDC-UCM y Catarata.

Ojeda, T. (2016). Relaciones internacionales y cooperación con enfoque Sur-Sur. Madrid: IUDC-UCM y Catarata.

Ojeda, T. (2015). La Agenda 2030 para el desarrollo sostenible en el marco de la cooperación Sur-Sur, en J. A., Sotillo, El reto de cambiar el mundo. $\mathrm{La}$ Agenda 2030 de desarrollo sostenible (pp. 96-102). Madrid: Editorial Catarata y ucM.

ONU. (17/09/2018). La función de la cooperación SurSur y la implementación de la Agenda 2030 para el Desarrollo Sostenible: retos y oportunidades. Informe del Secretario General, Nueva York: Asamblea General. https://bit.ly/2wmw10D

ONU. (10/07/2017). Marco de indicadores mundiales para los Objetivos de Desarrollo Sostenible y metas de la Agenda 2030 para el Desarrollo Sostenible. Labor de la Comisión de Estadística en relación con la Agenda 2030 para el Desarrollo Sostenible, Nueva York: Asamblea General. https://undocs. org/es/A/REs/71/313
ONU. (20/11/2016). Declaración de Quito sobre ciudadesy asentamientos urbanos sostenibles para todos. Ecuador: Conferencia de las Naciones Unidas sobre la Vivienda y el Desarrollo Urbano Sostenible (Hábitat III).

ONU. (2017). Nueva Agenda Urbana. Secretaría de Hábitat III. Ecuador. https://bit.ly/2h5SwKv

ORU-Fogar. (2018). Declaración de Rabat. XI Asamblea General del Foro Mundial de Regiones. 22 y 23 de septiembre. https://bit.ly/2y4M0MT

PNUD. (2019). Localización de la Agenda 2030 en México. Sistematización de la instalación y operacionalización de los Órganos de Seguimiento e Instrumentación de la Agenda 2030. México: PNuD. https:// bit.ly/2Hv7m3J

Thematic Research Network on Data and Statistics (TRendS). (2019). Metropolitan Localized Data in Greater Belo Horizonte, Brazil: A Participatory Guide to Better Governance. https://bit.ly/2GAQAYv un. (2019). Buenos Aires outcome document of the second High-level United Nations Conference on SouthSouth Cooperation. Disponible en https://undocs. org/pdf?symbol=en/A/CONF.235/3

\section{ENTREVISTAS}

Simón, Guadalupe. Asesora Técnica de Cooperación Internacional de la Representación de Relaciones Internacionales del Gobierno de la Provincia de Salta (Argentina). Entrevista personal realizada por vídeo llamada. 24/05/2019 y 29/05/2019.

Larrosa, Lucy. Coordinadora de proyectos de cooperación del Gobierno de la Provincia de Cerro Largo (Uruguay). Entrevista personal realizada por vídeo llamada. 10/05/2019 y 27/05/2019. 